\title{
EchoGéo
}

$20 \mid 2012$

Des mobilités aux transports. Regards croisés en Afrique de l'ouest

\section{Mondialisation et nouvelle mobilité des élites africaines}

La mobilité bourgeoise au Sud

Michel Lesourd

(2) OpenEdition

Journals

Édition électronique

URL : https://journals.openedition.org/echogeo/13118

DOI : $10.4000 /$ echogeo. 13118

ISSN : 1963-1197

Éditeur

Pôle de recherche pour l'organisation et la diffusion de l'information géographique (CNRS UMR 8586)

\section{Référence électronique}

Michel Lesourd, « Mondialisation et nouvelle mobilité des élites africaines », EchoGéo [En ligne], 20 | 2012, mis en ligne le 13 juillet 2012, consulté le 11 août 2021. URL : http://journals.openedition.org/ echogeo/13118; DOI : https://doi.org/10.4000/echogeo.13118

Ce document a été généré automatiquement le 11 août 2021.

EchoGéo est mis à disposition selon les termes de la licence Creative Commons Attribution - Pas d'Utilisation Commerciale - Pas de Modification 4.0 International (CC BY-NC-ND) 


\section{Mondialisation et nouvelle mobilité des élites africaines}

La mobilité bourgeoise au Sud

Michel Lesourd

1 Les dynamiques de la mondialisation sont variées. La simultanéité de l'avènement de la mondialisation des échanges, des communications et de la dérégulation des économies a créée pour les classes dirigeantes d'Afrique (haute fonction publique, élites politiques, chefs d'entreprise de l'industrie et des services, grands commerçants, responsables religieux et sociaux), des conditions particulièrement favorables à l'accumulation de richesses.

2 On mobilisera ici des concepts et notions qui ont, depuis longtemps, fait leurs preuves, et qu'il n'est pas nécessaire de présenter. Toutefois, l'on gardera à l'esprit qu'il s'agit de classifications commodes qui, en contexte africain, révèlent des contenus et des comportements pouvant différer quelque peu des modèles établis en Europe.Ainsi emploiera-t-on le terme élites pour désigner indifféremment les catégories sociales dominantes, détentrices de pouvoir, que celui-ci soit politique, économique ou socioculturel très diverses; bourgeoisie, pour des populations à niveau de vie supérieur (dix fois le salaire minimum?); classe moyenne pour caractériser une catégorie sociale intermédiaire, à réelle capacité de consommation, avec des loisirs. La Banque Mondiale considère que, sur le continent africain, il y aurait à peine 13 millions de riches (43 en 2030), mais que dans quelques pays, marqués par une croissance économique élevée, a émergé une classe moyenne beaucoup plus nombreuse : en Afrique du Sud, Nigeria, Ghana, Côte d'Ivoire, Sénégal, Kenya. Ceux qui constituent le "prochain milliard » (de consommateurs) ne gagnent qu'entre 2 et 10 US\$ par jour. Futurs consommateurs ou consommateurs " low cost ", ils se situent au-delà des problèmes de survie, mais très en deçà du seuil de consommation des élites consommatrices. On objectera que ces catégories ne sont guère cernées. Mais toutes, y compris la plus modeste, montrent une capacité graduée à consommer, et cela, en termes de mobilité spatiale, compte beaucoup. On ne s'intéressera pas aux plus riches, ces millionnaires nomades connus pour leur mode de vie de jet setteurs planétaires, mais à celles et ceux qui, par leur aisance 
relative, contribuent aux recompositions socio-spatiales de leur ville, de leur région, et à créer de nouvelles spatialités dans la mondialisation. En somme, des échelles renouvelées de spatialité des groupes sociaux, des familles et des individus.

4 Si le terme "traditionnel » est galvaudé, il fait clairement référence à un socle de valeurs ancrées dans le passé, ce qui n'empêche d'ailleurs en rien de le voir évoluer, la tradition étant d'abord cette interprétation-réinterprétation constante des savoirs, savoir-faire et règles d'une société confrontée au présent et à la préparation de l'avenir et puisant des références dans la connaissance d'un passé généralement normatif. On fera donc ici référence à des formes classiques anciennes de la richesse, présentes en Afrique aussi bien en milieu rural qu'en ville, autour des activités agricoles, pastorales, commerciales, d'autorité par la naissance et l'hérédité comme par les droits fonciers, et aussi religieuses. Ces élites politiques et religieuses, grandes familles maraboutiques et riches commerçants au Sénégal, grands agriculteurs et planteurs de Côte d'Ivoire, du Niger et du Cap-Vert, notables politiciens installés, forment des groupes hétérogènes de possédants dont la modernité ne remet en cause ni la capacité d'accumulation financière, ni la puissance matérielle, ni le prestige social.

Des informations obtenues par enquête participante auprès de familles cap-verdiennes et sénégalaises, étayée par divers écrits scientifiques, constituent la trame de base de cette recherche. Ces informations sont complétées par celles obtenues auprès d'ouvrages de recherche ou de synthèse, notamment pour le cas du Niger.

6 Sont également sous-jacentes la méthode d'analyse et l'approche sociologique qui insistent sur l'ostracisme social, l'entre-soi, l'importance accordée à l'éducation des enfants et d'autres marqueurs de la modernité de ces élites, comme le voyage ou la curiosité pour le cosmopolitisme. On pourrait donc, au-delà de l'intégration de la mobilité comme modus operandi socio-spatial familial et sociétal, s'interroger sur la capacité des élites nouvelles à s'organiser comme groupe social pour fonctionner et se maintenir comme groupe dirigeant, et à oeuvrer à l'intérêt général du pays où elle se trouve. Est-ce le cas? Il est permis d'en douter (Pinçon-Charlot et Pinçon-Charlot, 2010). D'une façon plus générale, on renverra également aux dynamiques des élites émergentes africaines (Lebeau et alii, 2003).

\section{Mise en place des classes moyennes et nouvelles conditions de la mobilité}

7 Dans une Afrique socio-économiquement profondément inégalitaire, l'ajustement structurel imposé aux États dans les années 1980 a favorisé, au moins localement, l'émergence d'une élite africaine qualifiée sur le modèle entrepreneurial occidental mais aussi sur des modèles rentier ou/et informel. Une classe moyenne de niveau supérieur, de hauts fonctionnaires et d'enseignants, de cadres d'entreprises, ingénieurs, gestionnaires, de responsables d'organismes de services, comme les ONG, de banquiers, s'est constituée au fil des décennies, à côté et avec les profiteurs de la rente de l'État.

Depuis le début des années 2000, la tendance lourde amorcée par les détenteurs de capitaux du monde entier, notamment ceux provenant du pétrole, de produits illicites, ainsi que le développement, dans certains pays, d'investissements tournés vers la résidence de séjour touristique, ont multiplié les placements fonciers et immobiliers 
spéculatifs dans les villes du Sud comme Dakar, Marrakech, Luanda ou encore Praia. Leurs disponibilités financières nouvelles et leur influence sociale leur ont permis de réaliser ce processus d'accumulation : c'est, sinon la naissance d'une classe moyenne aisée, comme en Inde, au moins la mise en place d'une élite africaine capable d'entrer dans le modèle de consommation occidental. Le Cap-Vert et le Sénégal, et dans une moindre mesure le Niger, connaissent depuis une décennie des taux élevés de croissance macro-économique, souvent supérieurs à $5 \%$ par an. La volonté des gouvernements de libéraliser l'économie et les politiques sectorielles menées ont favorisé l'émergence d'une petite élite commerçante, intellectuelle, financière, politique, capable d'accéder aux modes de consommation des pays développés.

Cette catégorie socio-économique nouvelle rejoint ainsi, en termes de capacité de consommation, les élites traditionnelles, notamment agricoles et commerçantes, mais aussi religieuses, historiquement constituées dans d'anciennes structures sociopolitiques incomplètement préservées.

10 Ces élites rurales sont connues : grands planteurs Agni du Moronou ivoirien, qui déjà, au temps du boom cacaoyer de la fin des années 70 , recevaient le visiteur en lui offrant du champagne de France (Gastellu, 1989). Les débouchés offerts à l'agriculture se sont multipliés, révélant de riches agriculteurs, tels ces grands producteurs-exportateurs d'oignons au Niger (Assagne Dagna, 2006). Ces élites rurales sont aujourd'hui renouvelées par les entrepreneurs en agriculture venus de la ville, qui investissent autant dans le périurbain que dans le rural profond, soutenues par l'essor de la consommation du vivrier marchand dans les villes.

On renverra au «bigmanisme " politique, adossé on non à la richesse foncière, phénomène ancien lui aussi, depuis Félix Houphouet-Boigny dans les années 1950, et les élites éduquées. Leur rôle est connu et l'on sait que ces élites ne sont pas un produit seulement colonial, qu'elles se sont dressées contre ce pouvoir colonial et ont joué un grand rôle dans la construction des États contemporains. Daloz (2002) insiste sur ces élites historiques qui, en leur temps, avaient intégré le dispositif colonial de mobilité éducative menant à la formation supérieure (dans le pays-métropole), qui ont été renouvelées après les indépendances par les stratégies des grandes familles désireuses d'utiliser la formation de type occidental pour asseoir leur position sociale. Plus récemment, des familles aristocratiques du Sénégal ont développé des stratégies comparables «d'investissement dans l'intelligence ». Elles conduisent certains jeunes à une importante mobilité pour études et à la constitution de diasporas familiales multicontinentales.

Enfin, les grandes familles religieuses, notamment en Afrique musulmane, constituent, depuis plus d'un demi-siècle, une élite originale, aujourd'hui largement engagée dans des processus de placement et de diversification de leurs ressources autrefois fondées seulement sur l'agriculture et le commerce.

On laissera de côté, en revanche, les multiples exemples de circulation professionnelle des élites commerçantes, qu'elles s'enracinent dans une tradition ancienne, comme les Al Hazaï du Niger (Grégoire et Labazée, 1993), qu'elles se développent dans le cadre d'opportunités plus actuelles, comme ces commerçantes mauritaniennes circulant entre Nouackchott et Paris, allant «faire » les supermarchés de Las Palmas et Dubaï, et, pour les plus riches d'entre elles, poussant jusqu'en Chine (C. Lesourd, 2006, citée par Choplin et Lombard, 2009), ou encore qu'elles s'inscrivent dans un «colportage transnational » contemporain (Tarrius, 2010). 

avec les volontés politiques des États et les stratégies sous-régionales visant à dynamiser les infrastructures et les acteurs du transport et répondant à la « révolution silencieuse " des transports (Lombard, 2011). Liées à ces logiques d'intérêt régional (CEDEAO, 2005), on fera également référence, au moins localement, à celles du développement touristique, qu'il s'agisse de tourisme familial ou d'affaires. Enfin, comment ne pas mettre en relation cette montée des élites et leur mobilité sans évoquer la relation puissante à l'urbanisation généralisée qui caractérise le continent depuis plus de quatre décennies et a abouti à la constitution de vastes métropoles abritant des populations allant des plus modestes aux plus aisées? aux comportements familiaux et individuels récents permis par la combinaison de nouveaux outils de mobilité dont disposent les unités familiales aisées, comme les voitures et l'accès financier au transport aérien. La dispersion géographique grandissante de la famille, devenue nationale, et même transnationale, conduit à la multiplication des déplacements. La combinaison cumulative de ressources financières moyennes ou élevées et du niveau d'éducation, d'information et de connaissance de pays étrangers acquise par la formation, la connexion numérique et la maîtrise de l'Internet, contribue à supporter les mobilités nouvelles.

Dans le contexte d'une Afrique mondialisée, la diffusion de multiples courants de pensée et de modes de vie influence des sociétés africaines de plus en plus ouvertes, multiculturelles ou/et transnationales. Ces influences se traduisent par le renouveau ou l'accroissement de la mobilité religieuse, par certaines pratiques plus affirmées (pèlerinages, tournées) ou la mobilité de loisir.

17 La mobilité résidentielle intra et périurbaine est rendue possible par l'accès à la deuxième voiture : l'habiter loin, dans une résidence fermée par exemple, s'accorde à une mobilité accrue dans le réseau intra-familial, une mobilité quotidienne de formation, une mobilité pratique pour la recherche de produits de consommation, une mobilité professionnelle.

Ces pratiques de mobilité, nouvelles ou renouvelées, sont soutenues par la volonté de continuer de respecter les fondamentaux des sociétés africaines : consolidation des réseaux familiaux et sociaux par des visites familiales à l'occasion des cérémonies d'initiation, religieuses, de décès surtout, des réunions de conseil de famille, de village, qui maintiennent les contacts entre la partie urbaine de la famille et la partie rurale, dans un continuum rural-urbain renforcé.

19 Ainsi, l'aisance financière, combinée aux influences portées par la mondialisation des échanges et de la communication-information, la vie urbaine et ses nouveaux métiers, le statut social ancien renouvelé, la permanence de certains fondamentaux sociaux, comme la famille, le rapport au village, la religion, semblent contribuer au développement et au renforcement de la mobilité des élites anciennes ou nouvelles et inciter à de nouvelles formes de mobilité. 


\section{Les lieux dans la ville : habiter, sortir, bouger}

20 La mobilité intra-urbaine des classes aisées se fait selon des repères spatiaux précis, mobilisés en termes de résidence, de but ou étape de sortie, de déplacement automobile.

\section{Habiter}

21 Les nouvelles bourgeoisies migrent vers des quartiers résidentiels. Elles contribuent à la création et au renforcement de la fragmentation, voire du cloisonnement sociourbain. Les quartiers résidentiels bien ventilés, aux vastes villas jardinées sont souvent situés à l'écart des anciens « Plateaux » : La Sablière à Libreville, Cocody Deux Plateaux à Abidjan, Palmarejo à Praia, Les Almadies, Fann, à Dakar, Ouaga 2000 à Ouagadougou. Ce sont, par exemple, les Gucci Revolutionaries, la nouvelle élite économique noire, qui s'installent dans la banlieue très résidentielle de Parktown North à Johannesburgh. On y développe à des degrés divers un entre-soi qui constitue un nouveau modèle sociospatial. Les manières d'habiter prennent des formes variées, notamment celle de véritables lotissements abritant des catégories professionnelles assez homogènes, de villas et d'habitat en immeubles résidentiels fermés, comme les condominiums.

Ces nouvelles manières d'habiter renvoient à la dynamique urbaine récente des grandes métropoles africaines. L'étalement urbain, connu surtout par les immenses quartiers spontanés périphériques, vaut aussi pour les quartiers les plus résidentiels. Ainsi, dès les années 80, accompagnant le boom cacaoyer, Abidjan a créé l'ultrarésidentiel Cocody Deux Plateaux pendant que, au nord de la ville naissait Abobo-gare. La décennie Wade, à Dakar, a vu s'étendre et se densifier le luxueux quartier de la Pointe des Almadies, mieux accessible grâce à l'aménagement de la route de la corniche. Au Cap-Vert, le volontarisme urbanistique créait de toutes pièces Palmarejo, à l'ouest de la ville de Praia (Medina do Nascimento, 2009).

Le cas des élites religieuses semble un peu différent. Dans les capitales religieuses, comme Touba ou Kaolack au Sénégal, respectivement capitale religieuse et ville de résidence des Khalifes des confréries Mouride et de la Tidjaniya Niassène, les élites religieuses traditionnelles sont depuis longtemps installées dans de véritables quartiers réservés à leur usage résidentiel. Ils disposent toutefois, avec les principaux membres de leur famille, de plusieurs autres résidences, notamment à Dakar, dans les quartiers de bon et très bon standing : des membres de la famille s'installent dans de vastes villas, point de départ de stratégies d'action religieuse et sociale au sein de ces quartiers. Au Sénégal, les familles les plus aisées esquissent ainsi un véritable réseau résidentiel inter-urbain. 
Illustration 1 - Salon de terrasse d'une villa de famille maraboutique. Tivaouane (Sénégal)

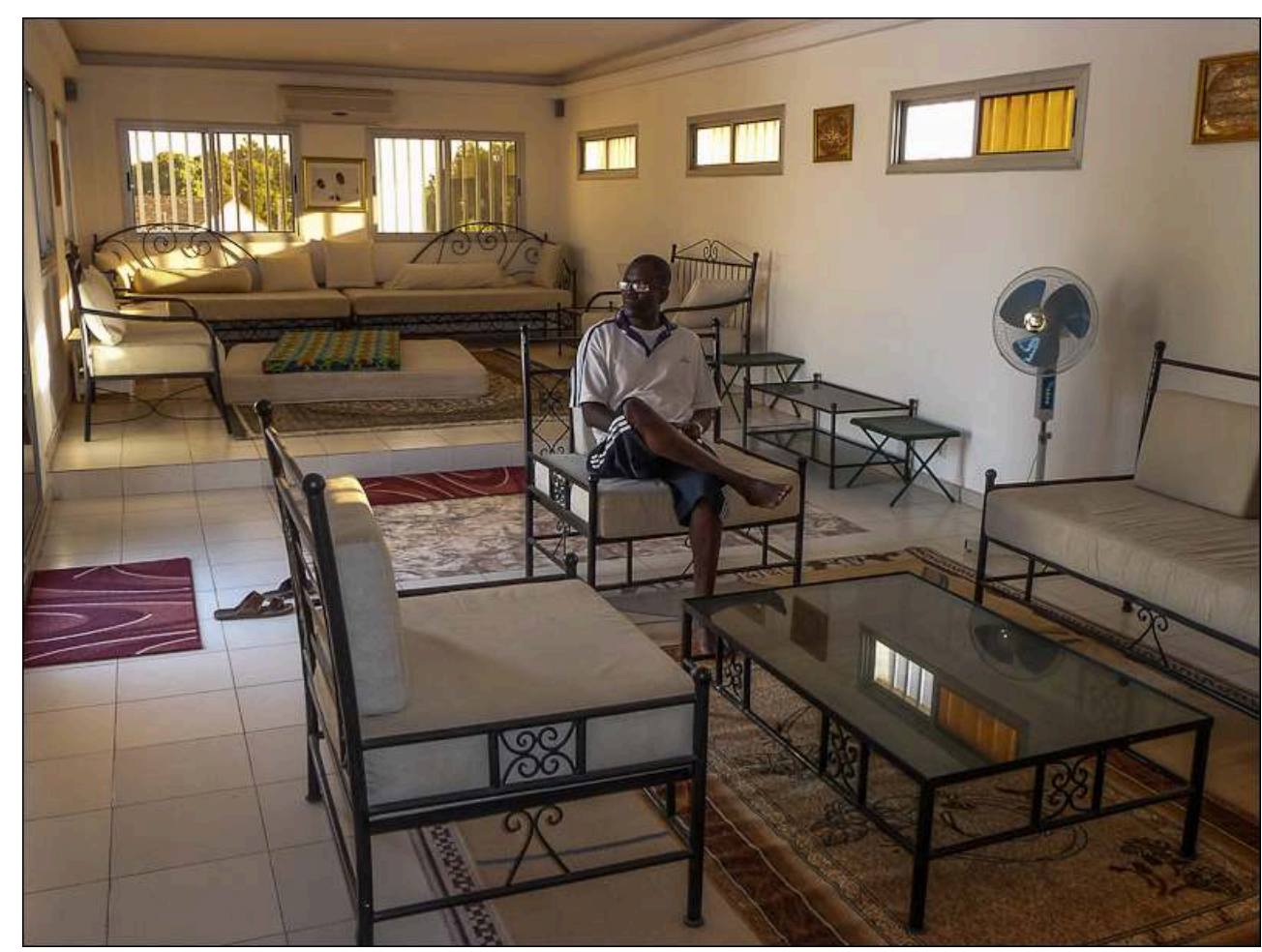

Une nouvelle manière d'habiter pour des activités traditionnelles : vastes pièces de réception pour la famille à l'occasion de fêtes et cérémonies religieuses impliquant un séjour long de personnes venant parfois d'outre-mer. L'aisance financière permet de financer les voyages des membres de la famille.

Auteur: Michel Lesourd.

\section{Sortir}

La consommation de loisir s'inscrit dans une géographie marquée par les nouveaux lieux centraux de la sociabilité des riches. Ce sont des restaurants de luxe, comme le Montparnasse ou La Croisette à Abidjan, des bars branchés, comme à Dakar où, au coeur du Plateau, le Café de Rome est devenu le lieu emblématique de cette nouvelle élite aisée. Ce sont des hôtels de luxe et les casinos - le Terrou Bi - à Dakar, des plages protégées, comme sur l'île Boulay face à Abidjan et sa "Baie des Milliardaires ». Et, depuis peu d'années, des salles de fitness, des salons de coiffure, les spas, les clubs avec piscine.

Les galeries commerciales esquissent une géographie plus affirmée des quartiers aisés de loisir-commerce et non plus seulement de résidence, comme l'a été longtemps celui de la Pointe des Almadies à Dakar. Ainsi la nouvelle Sea Plaza à Dakar $(1,4$ ha, 84 boutiques aux enseignes internationales, 6 fast food et glaciers, un restaurant, un bar lounge, un supermarché, bowling, garderie, spa), communicant avec le luxueux hôtel Radisson Blu sur la corniche de Fann, ou encore le Ghana Mall à Accra (2 ha, 1000 places de parking, 65 boutiques en galeries climatisées), ont-ils l'ambition d'initier de véritables zones de consommation d'un nouveau type, où luxe et détente séduisent non seulement le consommateur fonctionnel à la recherche d'un produit de qualité, mais également toute la famille. 


\section{Bouger} tapageuses limousines de l'élite bling bling, on constate que les classes moyennes disposent désormais d'un ou deux véhicules familiaux, ce qui autorise la multiplication des déplacements en famille ou pour la famille vers des écoles choisies, des sorties pizzas, glaciers, salons de thé, clubs, terrains ou salles de sport, piscines et plages, lieux-repères désormais intégrés aux loisirs des classes moyennes. Ainsi la géographie vécue de la ville se modifie-t-elle : une mobilité urbaine sans commune mesure avec le passé, pour les heureux bénéficiaires d'un véhicule, permet de créer ou de renforcer une dynamique inter-quartiers. Même si l'espace vécu du quartier construit par la maison, le voisinage et la vie de rue pouvait se construire en se passant de mobilité motorisée, cette spatialité augmentée s'accompagne de nouveaux repères, itinéraires, habitudes : une nouvelle territorialité, une façon nouvelle d'habiter la ville. En même temps, un autre lieu est devenu tout aussi emblématique de la mobilité : l'aéroport, qui concentre toutes les attentions, pour le voyage, pour l'accueil de retour des éléments de ces familles, éventuellement transnationales, de la société émergente: on s'y déplace en famille.

cette mobilité urbaine augmentée répond, dans les campagnes, une mobilité rurale élargie. On reprendra l'exemple des grands producteurs d'oignons du Niger. L'accroissement de leur pouvoir d'achat ainsi que celui des commerçants aisés a plusieurs conséquences sur leur mobilité locale individuelle et celle des membres de la famille :

- l'accès aux soins de santé, par la fréquentation d'un hôpital ou d'un centre de santé peu éloigné (Madoua au Niger). La mobilité ordinaire scolaire complète cette intégration de la consommation des services sociaux courants.

- la mobilité de loisir des jeunes, en bicyclette, en moto, entre le village des producteurs et les centres urbains, les parents faisant de même, mais avec une voiture. Ne faut-il pas souligner la vitalité de cette mobilité « banale » des jeunes de la campagne qui vont passer leurs loisirs au bourg voisin? L'enracinement dans les territoires, les réseaux de villages dominés par des lieux centraux que sont les bourgs et les petites villes semblent constituer, au moins dans des espaces ruraux densément peuplés, le signe d'une vitalité et d'une modernité rurale longtemps compromises.

- les déplacements locaux et régionaux des chefs de famille sont renforcés par leur implication dans les organisations socioprofessonnelles locales, le courtage et les projets de développement. L'aisance des élites locales contribue donc à faire vivre la décentralisation et la communalisation des territoires.

\section{La mobilité traditionnelle renouvelée}

Les déplacements traditionnels obligés par des relations familiales étendues et toujours prégnantes (décès, fêtes, conseils de famille) demeurent, localement et nationalement, un fait fondamental, auquel s'ajoutent, avec une dimension plus internationale, des déplacements accrus de caractère religieux (notamment pour les adeptes des religions à pèlerinage). 


\section{Déplacements familiaux}

L'exode rural n'a pas tari l'importance du village comme lieu de rencontre, de maintien de la présence familiale au sein de la société rurale, de ressourcement. Il convient de continuer d'être présent pour chacun des évènements cruciaux de la vie de la communauté clanique, villageoise, pluri-villageoise, et ce d'autant plus que l'on occupe une position importante à la ville. Même si leur diversité contribue à la fréquence des déplacements, c'est avant tout d'obligations sociales qu'il s'agit. La distance (distancetemps surtout) compte peu au regard de la nécessaire présence, qu'il s'agisse de participation à des décisions impliquant toute la communauté territoriale ou d'évènements d'ordre privé familial. Ainsi, tel universitaire dakarois chef de famille, vivant à près de 9 heures de taxi-brousse de son village natal du Fouta Toro, fera un aller-retour express à peine le temps d'un week-end (18 heures de voyage aller-retour) pour une fin d'après-midi et une unique soirée de discussion familiale consacrée à des affaires financières et religieuses.

Le mariage demeure une institution sociale majeure dans les sociétés chrétiennes et musulmanes. Au Cap-Vert, pays catholique romain à $98 \%$, le mariage est l'occasion d'affirmer ou de réaffirmer l'union de deux familles. Malgré ou peut-être à cause d'un développement important de l'union libre et des familles monoparentales où la mère élève le plus souvent seule ses enfants, le mariage prend une importance particulière aux yeux des familles comme garant de l'ordre social dominant des élites du pays : si le mariage est une affaire de familles croyantes, il est aussi peut-être, dans ce pays, affaire d'affirmation sociale. Les fêtes, alors, sont fastueuses, et les déplacements massifs des différentes familles vers les lieux de la cérémonie, la campagne comme la ville, attestent de la solidité des liens.

\section{Une forme de mobilité traditionnelle renouvelée : le pèlerinage}

31 Les disponibilités financières dont disposent les bourgeoisies rurales et urbaines permettent, en pays musulman, de réaliser le pèlerinage aux lieux saints de l'islam, l'une des obligations caractéristiques de mobilité de tout fidèle de cette religion. Ainsi peut-on constater, en Afrique sub-saharienne, un essor récent de cette pratique, autrefois réservée à de rares élites aristocratiques et aux chefs des familles maraboutiques. Le pèlerinage est l'expression manifeste de la piété. Il est une marque d'identité sociale. Les pèlerinages sont des déplacements de prestige, très coûteux, mais chargés d'un capital culturel et symbolique considérable.

Chez les grands producteurs d'oignons de la région de Tahoua au Niger, la mobilité est liée à des stratégies familiales et sociales précises qui combinent satisfaction des besoins fondamentaux et surplus comme «investissement social», comme «transformation du capital économique en capital social » (Assagne Dagna, op. cit, p. 184), modèle que l'on retrouve chez les grands commerçants de Maradi et Zinder. L'investissement par le don et le partage liés au pèlerinage est aussi un investissement symbolique dans la famille paternelle, utérine, et la clientèle.

33 Le pèlerinage à La Mecque, dans un pays à $98 \%$ musulman, est évidemment souhaité pour des raisons religieuses. Mais la cause sociale n'est pas moindre. Le titre de hadj confère à celui qui le détient un rang social élevé. Il est clairement un indicateur d'un pouvoir financier élevé, de l'ordre de vingt à cinquante fois supérieur à celui d'un petit 
producteur. Cette mobilité socioreligieuse est comparable à celle des grands commerçants Al Hazaï qui font des voyages d'affaire à Dubaï et pratiquent la oumra, le petit pèlerinage, tout au long de l'année. Les producteurs d'oignons les plus aisés font, eux, plusieurs fois le grand pèlerinage : 3 à 4 selon Assane Dagna en l'espace de 10 à 15 ans. Ils se rendent seuls aux lieux saints, et non avec leur(s) épouse(s) : c'est en effet au fils aîné, devenu, plus tard, un paysan aisé, que revient l'honneur d'offrir le voyage à sa mère.

Dans un tout autre contexte, un exemple significatif de cette néo-bourgeoisie est celui de cette famille sénégalaise vivant et travaillant à Dakar. Elle est constituée d'un père professeur d'enseignement supérieur et son épouse ingénieure, avec trois enfants. Les parents sont tous deux descendants de vieilles familles aristocratiques. En 2009, la mère de l'épouse, qui souhaite faire son pèlerinage, est âgée. Aussi sa fille décide-t-elle de l'accompagner. L'année suivante c'est le mari, chef de famille, qui prend la décision de faire, à son tour, le grand pèlerinage. Même si l'organisation est depuis longtemps rôdée depuis le Sénégal, avec des vols directs Dakar- Djeddah, le déplacement entraîne des frais considérables pour la famille, une absence de plus d'un mois et une indisponibilité relative, au retour de plusieurs semaines, qui n'est pas sans incidence sur les activités professionnelles du pèlerin. Les dimensions sociale, pour la famille, les amis et la parentèle, et spirituelle, plus personnelle, sont évidentes. Comme dans le cas des grands planteurs d'oignons du Niger, la mobilité s'inscrit ici dans la sphère collective autant que familiale, comme un marqueur social.

\section{Déplacements de fin de semaine et voyage combiné}

Deux types de comportements nouveaux sont clairement identifiés. Ils témoignent de la modernité des modes de vie et de consommation revendiquée par cette nouvelle génération bourgeoise, ouverte à des comportements que d'aucuns jugent être occidentaux. Ce sont les déplacements et la circulation de fin de semaine, et le voyage lointain d'agrément.

\section{Les déplacements de loisir de fin de semaine et de fête}

Généralement familiaux, dans des espaces résidentiels proches, ils sont devenus une réalité banale. Ainsi, la plage ou la montagne constituent-ils des lieux de rencontre, de séjour d'un après-midi ou du week-end. Ils s'accompagnent de nouveaux comportements. La baignade, y compris en respectant des codes vestimentaires non européens (pour les familles musulmanes), le déjeuner au restaurant, la promenade, deviennent la norme. 
Illustration 2 - Une auberge de montagne pour la fin de semaine : Rui Vaz, Santiago, Cap-Vert

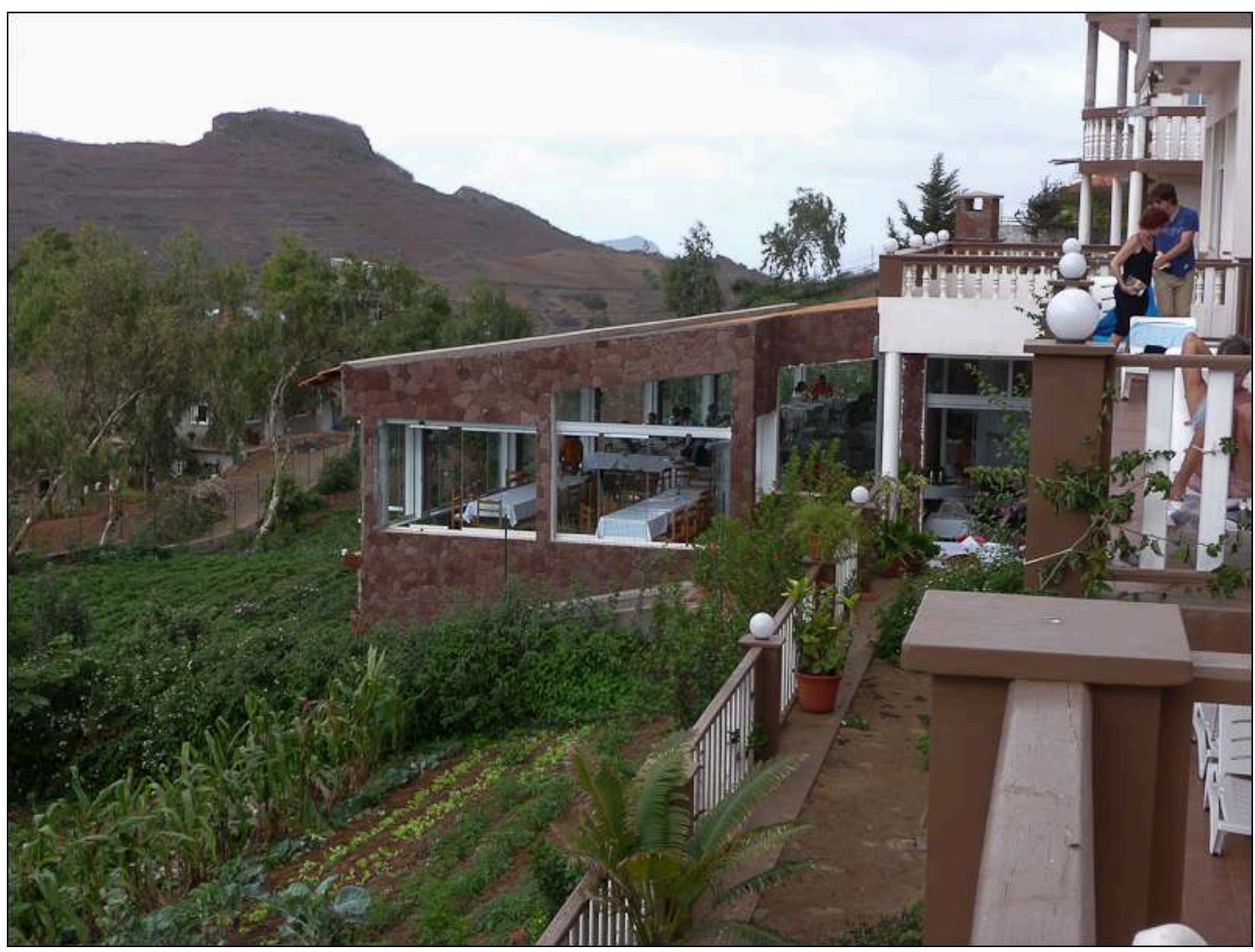

Repas familiaux et amicaux et, plus rarement, location de chambre dans une pousada de gamme moyenne, afin de profiter de la fraîcheur de la montagne.

Auteur : Michel Lesourd.

Dans l'île de Santiago du Cap-Vert, la fréquentation par la bourgeoisie de Praia de quelques lieux situés à moins d'une heure de voiture, combinant beautés naturelles et restauration de qualité, témoigne de cette approche nouvelle du loisir. L'auberge de Rui Vaz, à près de $1000 \mathrm{~m}$ d'altitude, les petites villes balnéaires de Praia baixo et Tarrafal sont les plus fréquentées. A une heure de route de Praia, les familles visitent aussi, comme une promenade forestière éducative, le parc naturel d'altitude de la Serra Malagueta.

38 Au Sénégal, le fait balnéaire s'est considérablement développé depuis la fin des années 90, certes en raison de la proximité des plages dakaroises, mais surtout parce que la métropole ouest-africaine offre des services de clubs nautiques de qualité avec des piscines-restaurants qui permettent aux familles de passer des moments de détente. Mais il faut la voiture de famille ou le taxi pour y accéder. Saly, autre espace plus lointain, à deux heures de route, accueille les familles désireuses de passer une fête ou un week-end de détente balnéaire. Les plus aisés des dakarois y trouvent une gamme variée de réceptifs. Lors des fêtes de Noël et de fin d'année, les familles prennent la route et s'installent pour deux ou trois nuits dans les hôtels bordant la plage.

\section{Le voyage combiné}

Les nouvelles mobilités bourgeoises s'expriment aussi sous la forme du voyage combinant l'agrément (balnéaire, loisirs hivernaux, séjour culturel urbain) avec des visites familiales mais aussi, tenant compte des différentiels de prix, des préoccupations d'approvisionnement à usage privé ou même commercial. C'est plutôt 
dans les sociétés à forte émigration internationale, comme les sociétés cap-verdienne ou sénégalaise, que ce modèle se développe, en relation avec la dynamique de transnationalisation des familles.

On citera l'exemple de ce couple cap-verdien vivant à Praia, capitale du Cap-Vert. Elle, cadre dans une entreprise privée, lui, ingénieur et cadre supérieur. Avec leurs deux enfants, ils sont partis passer les fêtes de fin d'année, en France pour quelques jours de sports d'hiver avec des amis français, puis à Lisbonne, au Portugal, pour saluer la branche de la famille qui y réside depuis plus de deux décennies. Si le voyage était d'abord un voyage d'agrément, il avait aussi un but familial, et il n'était pas exempt de préoccupations commerciales, puisque le couple a beaucoup acheté à Marseille et à Lisbonne.

41 Dans les familles transnationales, ces déplacements de loisirs prennent l'allure de véritables tournées familiales. On fera référence ici à un modèle fréquent de famille transnationale cap-verdienne éclatée sur trois continents et une demi-douzaine de pays, avec trois générations d'émigrés (début de l'émigration familiale : 1961), donc des cousinages au deuxième degré et l'accroissement de la parentèle avec les familles alliées par mariage de la deuxième génération. Au total, près d'une centaine de personnes réparties en 12 ménages émigrés : trois au Sénégal, trois aux USA, deux en France et au Portugal, un au Luxembourg et aux Pays Bas, sans oublier les six familles au Cap-Vert.

Parmi ces dernières, prenons le cas de cette famille de classe moyenne aisée : les deux parents travaillent, père cadre supérieur dans une entreprise privée, deux enfants de 16 et 13 ans, une maison de ville avec cuisine équipée, 2 salons, 4 chambres, buanderiecuisine, terrasse, la propriété d'un local commercial mis en location, un équipement informatique comprenant 1 ordinateur de bureau, 2 ordinateurs portables, 2 TV, téléphone fixe, 4 téléphones portables, des appareils de musique, deux voitures. Le ménage gagne quarante fois le salaire minimum cap-verdien.

En août 2010, le ménage a effectué un voyage familial et touristique d'un mois aux Etats-Unis. Deux frères de la mère habitent Pawtucket (Massachussets). Mais les familles ont visité Providence (Rhode Island), New Bedford, Boston, Cape Cod et le Maine, New York City.

Les conséquences de la spatialité transnationale de familles élargies aisées sur la mobilité individuelle de ses membres sont importantes. Les jeunes circulent davantage. Les raisons avancées sont la volonté de maintenir les liens de cousinage, profiter de la famille pour des séjours à l'étranger où l'on ne paye que le billet d'avion, favoriser la pratique des langues étrangères, et plus largement éduquer. Les adultes, eux, pensent au repos et aux loisirs, aux liens familiaux. Ils se montrent également très pragmatiques dans deux domaines : les soins médicaux et l'approvisionnement en produits introuvables ou très coûteux au Cap-Vert. Ainsi, cette famille cap-verdienne témoin, avec son séjour aux USA en août 2010 fit des achats de plus de 1500 dollars de produits alimentaires US et plus de 800 dollars de vêtements, chaussures et produits d'hygiène et santé, sans compter tous les équipements scolaires pour une année complète, et les présents prévus pour la famille ... Ces marchandises, non destinées à la revente, ont été transportées pour une part dans les bagages des voyageurs, mais surtout dans des «bidons» (illustration 3), ces petits conteneurs familiaux capverdiens qui, depuis des décennies, sont expédiés par cargo depuis New Bedford par la ligne régulière mensuelle à destination du port de Praia. Malgré le dédouanement 
obligatoire, l'affaire reste très rentable. Sur la «culture du bidon " au Cap-Vert, on renverra le lecteur à l'analyse très détaillée qu'en font $\mathrm{S}$. Bredeloup et J. Lombard (2008) comme technique de transport à propos de la crise du commerce de la fripe et, d'une manière plus large, comme moyen de ravitaillement de tout un peuple en produits venus de l'outre-mer.

Illustration 3 - Le voyage combiné : les bidons du Cap Vert présents jusque dans les salons et les chambres des maisons particulières

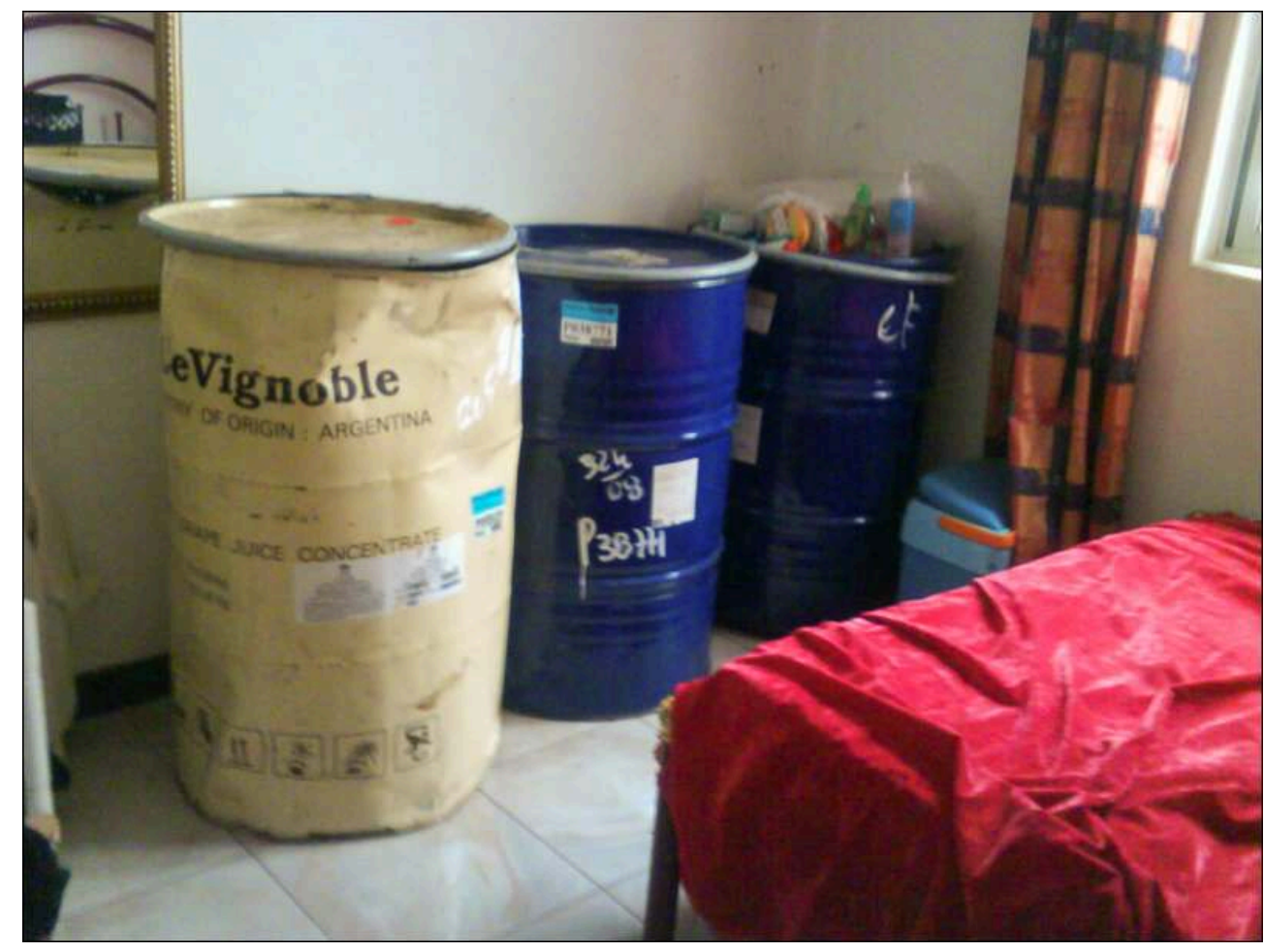

Auteur : Jérôme Lombard.

\section{Mobilité virtuelle et nouvelle spatialité de la famille connectée}

On connaît depuis longtemps la manière dont les migrants utilisent les TIC comme composante de leur mobilité (Lesourd, 2011). Dans les familles transnationales, le lien entre les différentes cellules du réseau familial éclaté est renforcé par la communication constante que ces dernières entretiennent entre elles.

Les activités communicationnelles sont, dans ces familles transnationales, particulièrement importantes. La famille est géographiquement éclatée mais jamais ses membres n'ont été aussi proches, "trans-rassemblés ». Dans la famille évoquée supra, Internet, Skype, Messenger, le téléphone, sont très mobilisés. La communication avec les frères et soeurs et cousins des États-Unis est quasi quotidienne. Avec Skype, on se voit. Elle est facilitée par les gadgets informatiques comme ce fameux "Magic jack » américain, acheté pour quelques dollars à Boston, et couplé à un abonnement international (20 dollars) pris aux USA qui permet ensuite, une fois rentré au Cap Vert, de communiquer sans frais avec la famille aux États-Unis. 

éducative, puisqu'elle fait découvrir d'autres manières de vivre, de se nourrir, de se familiariser avec des produits cosmopolites pour la plupart américains et européens.

\section{Conclusion}

La contribution des riches d'Afrique engendre de nouvelles spatialités et consommations d'espace. La mobilité combine le local avec le mondial, l'agréable à l'utile, le devoir au loisir : un nouveau modèle de circulation dans un monde en recomposition et une contribution originale à la nomadisation planétaire ? La tendance de "l'habiter" semble renforcer la différenciation ségrégative. Les consommations valorisent les réseaux sociaux, les lieux de loisir, et les pôles culturels plus conventionnels.

49 Ces nouvelles manières d'habiter et d'occuper les territoires renvoient à la question des stratégies de consolidation des pouvoirs de ces élites, tant à l'échelle locale que dans la mondialisation, leur choix d'une culture cosmopolite (ou non), pour préparer l'avenir et la réussite de leurs enfants. La nouveauté, en matière de circulation, ne vient pas des élites politiques telles qu'elles se sont développées dans les cinquante dernières années, avec leurs semis de résidences dans les métropoles mondiales, mais des classes moyennes supérieures, gagnées à des stratégies mondialisées d'éducation et de « voyager utile».

On comprendra aussi que la présentation de ces exemples ne reflète sans doute qu'une petite partie de la diversité des comportements des nouvelles élites, surtout de cette nébuleuse que constituent ces classes moyennes supérieures. Peut-on parler d'un nouveau groupe social de familles bourgeoises connectées? Et de riches familles (éventuellement transnationales) connectées ayant la mobilité en partage ? Peut-être, en ce qui concerne certains modes de consommation, et la mobilité en est un. Mais les stratégies et les comportements demeurent très divers, les idéologies qui sous-tendent le comportement de mobilité sont variées, et les intérêts divergents.

\section{BIBLIOGRAPHIE}

Assagne Dagna M.Les effets de la réappropriation de la culture du "Violet de Galmi » par les producteurs d'oignon de la région de Tahoua (Niger) sur la dynamique du territoire local, l'organisation sociale et économique. Thèse de doctorat, Université de Toulouse Le Mirail, $274 \mathrm{p}$.

Blanchet G., 1975.Elites et changements économiques et sociaux dans une perspective africaine. Publications du Centre ORSTOM de Dakar (Sénégal), 118 p.

Bredeloup S., Lombard J., 2008.Mort de la fripe en Afrique ou fin d'un cycle? Revue Tiers Monde, $\mathrm{n}^{\circ}$ 194, avr-juin 2008, p. 1-22.

EchoGéo, 20 | 2012 
Chéneau-Loquay A.(sous la dir. de), 2004. Mondialisation et technologies de la communication en Afrique. Paris, Karthala - Bordeaux, MSHA.

CEDEAO. Atlas régional des transports et des télécommunications dans la CEDEAO. Issy les Moulineaux, Club du Sahel et de l'Afrique de l'Ouest.

Choplin A., Lombard J., 2009. La Mauritanie offshore. Extraversion économique, État et sphères dirigeantes. Politique Africaine, $\mathrm{n}^{\circ} 114$, Dossier Mauritanie, au coup par coup, p. 87-104.

Daloz, J.-P., 2002. Élites et représentations politiques. La culture de l'échange inégal au Nigeria. Pessac, Presses universitaires de Bordeaux, ,174 p.

Fourchard L., Mary A., Otayek R. (dir), 2005. Entreprises religieuses transnationales en Afrique de l'Ouest. Paris, Karthala/IFRA, 537 p.

Gastellu J-M., 1989. Riches paysans de Côte d'Ivoire. Paris, L'harmattan, 178 p.

Grégoire E., Labazée P. 1993. Grands commerçants d'Afrique de l'Ouest. Logiques et pratiques d'un groupe d'hommes d'affaires contemporains. Paris, Karthala-ORSTOM, 262 p.

Lebeau Y., Niane B., Pirou A., de Saint-Martin M. (dir.), 2003. État et acteurs émergents en Afrique. Paris, Karthala, IFRA, $351 \mathrm{p}$.

Lesourd C., 2006. "Au bonheur des Dames ». Femmes d'affaires mauritaniennes de nos jours. Thèse d'anthropologie, Paris, EHESS.

Lesourd M., 2011. Traces coloniales. Le « Blanc » et « l'indigène », regards-traces croisés dans la mondialisation. In B. Galinon Mélénec (coord), L'Homme trace, Paris, CNRS, p. 305-330.

Lombard J., 2011. Transports d'ici, transports d'ailleurs : Du Sénégal à l'Afrique de l'Ouest. Dossier d'habilitation à diriger des recherches, Le Havre, 3 vol., vol. 3, 235 p.

Pinçon-Charlot M., Pinçon-Charlot M., 2010. Les ghettos du Gotha. Points/Seuil, Paris.

Tarrius A. 2010. Les nouveaux cosmopolitismes migratoires d'une mondialisation par le bas. In Bancel N., Bernault F., Blanchard P. et alii (ss la dir. de), Ruptures postcoloniales. Les nouveaux visages de la société française, La Découverte, Paris, p. 414-428.

\section{RÉSUMÉS}

Today, the social class of "leaders" in Africa (high public official, political elite, business managers, big traders) is the main benefiter of the wealth accumulation process that has been created by the globalization, the development of exchanges and communication, as well as the global economic deregulation. Since the 2000's, some capital holders investments and local touristic policies have significantly increased land speculation and real estate growth in several "South" cities such as Dakar, Marrakesh, Luanda or Praia. In spite of increasing social inequalities in the economic context marked by structural adjustment since the 1980s, an African elite has raised on the basis of entrepreneurship either formal or informal. Their financial capacities and their social influence allowed them to achieve this accumulation process. This new African elite class cannot be compared to the Indian middle-upper consumption class but is nevertheless aspiring for the western consumption standards.

These "nouveaux riches" take part in the global nomadic world but the observation of their behaviour shows that these "new nomads" are actually combining different kinds of mobility, for different purposes such as traditional commitments, social relationships, business, leisure, and consumption. Several effects of this phenomenon can be observed. In the big cities, the new bourgeoisie migrates to living districts, contributing to the intensification of urban social 
division. The traditional and social commitments (due to the funerals, the celebrations, etc.) remain a major purpose of local, national or international mobility and the religious purpose (especially for pilgrimage) has been significantly increasing. Two new behaviours can be identified. The first is the leisure trip of the weekends, generally in the local area with all the family. The second one is the trip combining different purposes: social visit or tourism during a professional trip, commercial operation combined to a family visit, etc. This kind of journey is particularly frequent in the societies that have a tradition of international emigration like Senegalese and Cap Verdean societies.

The contribution of African elites to the nomadic planet is thus complex, because it combines the local place with the world movements, the pleasant to the useful, and the duty in the leisure. Is it a new model of circulation in a world and an original contribution to the globalisation? We shall lean more specifically on Senegalese, Cape Verdean, Moroccan examples.

INDEX

Thèmes : Sur le Champ - Sur le Terrain

\section{AUTEUR}

\section{MICHEL LESOURD}

Michel Lesourd, Michel.Lesourd@univ-rouen.fr, est Professeur des Universités à l'Université de Rouen et membre de l'UMR CNRS 6266 IDEES/GéoSUDS. 